\title{
Lesões causadas pelo uso de cigarro eletrônico: revisão integrativa
}

\section{Injuries caused by the use of electronic cigarettes: an integrative review \\ Lesiones causadas por el uso de cigarrillos electrónicos: una revisión integradora}

Barbara Beatriz Lira da Silva

ORCID: https://orcid.org/0000-0001-9166-6147 Universidade Estadual do Piauí, Brasil E-mail: brbeatriz16@gmail.com

Ana Klara Rodrigues Alves

ORCID: https://orcid.org/0000-0002-1216-9386 Universidade Estadual do Piauí, Brasil E-mail: klaraphb@outlook.com

Lívia Filomena Castelo Branco Machado ORCID: https://orcid.org/0000-0001-5073-8140 Centro Universitário UNINOVAFAPI, Brasil E-mail: liviafilomenacbm@hotmail.com

Edmar José Fortes Júnior

ORCID: https://orcid.org/0000-0002-0114-9937 Faculdade de Ciências Humanas, Exatas e da Saúde do Piauí, Brasil E-mail: edmarfortes@hotmail.com

Cindy Moura Dias de Araújo ORCID: https://orcid.org/0000-0002-4902-3516 Faculdade de Ciências Humanas, Exatas e da Saúde do Piauí, Brasil E-mail: cindydiaasma@outlook.com

Mariana Fagan Peyrot

ORCID: https://orcid.org/0000-0003-3366-1350 Faculdade de Ciências Humanas, Exatas e da Saúde do Piauí, Brasil E-mail: marianapeyrot@gmail.com

Pâmela de Sousa Melo

ORCID: https://orcid.org/0000-0002-1564-6758

Centro Universitário UNINOVAFAPI, Brasil E-mail: pamela.melos97@gmail.com

Iluska Guimarães Rodrigues ORCID: https://orcid.org/0000-0003-2966-9018 Centro Universitário UNINOVAFAPI, Brasil E-mail: iluska.guimaraes@gmail.com

Luiza Emiliana Queiroz Bucar ORCID: https://orcid.org/0000-0003-4236-8439 Centro Universitário UNINOVAFAPI, Brasil E-mail: luizabucar@outlook.com

Camila Kizzy Trindade Oliveira ORCID: https://orcid.org/0000-0003-3391-2439 Centro Universitário UNINOVAFAPI, Brasil E-mail: camilakizzyto@gmail.com

Danielle Sotero Fortes Carvalho ORCID: https://orcid.org/0000-0002-1563-2946 Centro Universitário UNINOVAFAPI, Brasil

E-mail: dani_fortes1995@hotmail.com

Maria Clara Martins Costa

ORCID: https://orcid.org/0000-0002-6264-4069 Centro Universitário UNINOVAFAPI, Brasil E-mail: myrianclara@hotmail.com

Lucas Ferrari da Silva Mendes

ORCID: https://orcid.org/0000-0001-5904-839X Centro Universitário UNINOVAFAPI, Brasil

E-mail: lucasferrari.med@gmail.com

Victor Rocha Santos

ORCID: https://orcid.org/0000-0002-8068-3264 Centro Universitário UNINOVAFAPI, Brasil

E-mail: victoroxas@gmail.com

Sara de Queiroz Costa

ORCID: https://orcid.org/0000-0002-0070-8573 Faculdade Morgana Potrich, Brasil E-mail: saraqueirozcostaa@gmail.com 


\begin{abstract}
Resumo
Milhares de usuários de cigarros eletrônicos, predominantemente homens com idade entre 13 e 34 anos, desenvolveram sintomas respiratórios, gastrointestinais e sistêmicos após a vaporização. Visto isso, o presente estudo tem como objetivo descrever as consequências e lesões do uso de cigarros eletrônicos. Trata-se de um estudo de abordagem qualitativa, onde se adotou a revisão integrativa da literatura, que conforme Galvão (2012), é uma construção de uma análise ampla da literatura com passos pré-definidos uma vez que ela contribui para o processo de sistematização e análise dos resultados, visando a compreensão de determinado tema, a partir de outros estudos independentes. Realizado através da Biblioteca Virtual de Saúde (BVS) nas bases de dados do Pubmed e Medline, usando os cruzamentos dos descritores em inglês "Electronic cigarette", "Consequences", "Vaping". Os danos do CE à saúde cardiovascular mostram que os consumidores dos dispositivos estão mais expostos ao infarto agudo do miocárdio e doenças cerebrovasculares. O consumo de CE tem sido associado ao aumento da resistência das vias aéreas periféricas e da fração de óxido nítrico exalado, com características semelhantes aos efeitos da fumaça do tabaco, e à lesão pulmonar associada ao uso de produtos de CE. Além disso, a maioria dos jovens usuários de CE relata o uso de politabaco. Os cigarros eletrônicos e seus tóxicos liberados parecem prejudiciais a vários sistemas orgânicos, embora o corpo de evidências atual seja limitado, especialmente em termos de efeitos de longo prazo. Mais pesquisas são necessárias com foco em nas suas consequências, constituintes do e-líquido, características do usuário e padrões de uso.
\end{abstract}

Palavras-chave: Cigarro eletrônico; Saúde; Nicotina.

\begin{abstract}
Thousands of users of electronic cigarettes, predominantly men aged between 13 and 34, have developed respiratory, gastrointestinal and systemic symptoms after vaporization. Based on that, this study has as objective to describe the consequences and injuries of the use of electronic cigarettes. This is a study with a qualitative approach, which adopted an integrative literature review, which, according to Galvão (2012), is a construction of a broad literature analysis using pre-defined steps as it contributes to the systematization process and analysis of the results, aiming to comprehend an specific theme, based on other independent studies. It was developed through the Virtual Health Library (VHL) in the Pubmed and Medline databases, using the crossings of the descriptors in English "Electronic cigarette", "Consequences", and "Vaping". The damage of EC to cardiovascular health shows that users of those devices are more exposed to acute myocardial infarction and brain vascular diseases. The use of EC has been associated with increased peripheral airway resistance and exhaled nitric oxide fraction, with characteristics similar to the effects of tobacco smoke, and with lung damage associated with the use of EC products. In addition, most young EC users have reported the use of polytobacco. Electronic cigarettes and their released toxics seem harmful to many organ systems, although the current body of evidence is limited, especially in terms of long-term effects. More researches are needed focusing on its consequences, constituents of the e-liquid, user characteristics and usage patterns.
\end{abstract}

Keywords: Electronic cigarette; Health; Nicotine.

\title{
Resumen
}

Miles de usuarios de cigarrillos electrónicos, predominantemente hombres de entre 13 y 34 años, desarrollaron síntomas respiratorios, gastrointestinales y sistémicos después de la vaporización. Por ello, el presente estudio tiene como objetivo describir las consecuencias y lesiones del uso de cigarrillos electrónicos. Se trata de un estudio con enfoque cualitativo, que adoptó una revisión integradora de la literatura, que, según Galvão (2012), es una construcción de un análisis amplio de la literatura con pasos predefinidos, ya que contribuye al proceso de sistematización y análisis de los resultados, con el objetivo de comprender un tema determinado, a partir de otros estudios independientes. Realizado a través de la Virtual Health Library (BVS) en las bases de datos de Pubmed y Medline, utilizando los cruces de los descriptores en inglés "Electronic cigarette", "Consequences", "Vaping". El daño de la EC a la salud cardiovascular muestra que los usuarios de dispositivos están más expuestos a infarto agudo de miocardio y enfermedades cerebrovasculares. El uso de AE se ha asociado con una mayor resistencia de las vías respiratorias periféricas y la fracción de óxido nítrico exhalado, con características similares a los efectos del humo del tabaco y con daño pulmonar asociado con el uso de productos de AE. Además, la mayoría de los consumidores jóvenes de AE informan que consumen politabaco. Los cigarrillos electrónicos y sus tóxicos liberados parecen dañinos para muchos sistemas de órganos, aunque la evidencia actual es limitada, especialmente en términos de efectos a largo plazo. Se necesita más investigación que se centre en sus consecuencias, los componentes del elíquido, las características del usuario y los patrones de uso.

Palabras clave: Cigarro electronico; Salud; Nicotina.

\section{Introdução}

O tabagismo é a principal causa de doenças não transmissíveis em todo o mundo e é um importante fator de risco para doenças cardiovasculares (DCV) e doenças pulmonares. É importante ressaltar que dados recentes da Organização Mundial da 
Saúde (OMS) indicam que nas últimas duas décadas o uso global do tabaco caiu significativamente, o que foi em grande parte impulsionado pela diminuição do número de mulheres fumantes. Apesar desses avanços, o uso de cigarros eletrônicos (CEs) é uma tendência emergente, principalmente entre as gerações mais jovens. Há cada vez mais evidências de que os cigarros eletrônicos não são uma alternativa livre de danos aos cigarros de tabaco e há um debate considerável sobre se os cigarros eletrônicos estão salvando os fumantes ou gerando novos dependentes (Münzel et al., 2020).

CEs são dispositivos eletrônicos, consistindo essencialmente em um cartucho, preenchido com um líquido, um elemento de aquecimento/atomizador necessário para aquecer para criar um vapor que pode ser inalado através de um bocal e uma bateria recarregável. Tanto os dispositivos eletrônicos quanto os diferentes líquidos estão facilmente disponíveis em lojas ou lojas online. O líquido normalmente contém umectantes e aromatizantes, com ou sem nicotina; uma vez vaporizado pelo atomizador, o aerossol (vapor) proporciona uma sensação semelhante ao fumo do tabaco, mas supostamente sem efeitos prejudiciais. No entanto, foi relatado que o processo de aquecimento pode levar à geração de novos compostos de decomposição que podem ser perigosos (Burt \& Li, 2020; Marques, Piqueras \& Sanz, 2021).

Vaporizar nicotina ou usar cigarros eletrônicos tornou-se um fenômeno global popular nos últimos anos. Em comparação com 2011, onde 7 milhões de pessoas em todo o mundo usaram cigarros eletrônicos, o número de usuários de cigarros eletrônicos aumentou para 41 milhões em 2018, com jovens e adultos jovens representando o público que mais utiliza os cigarros eletrônicos (Struik \& Yang, 2021).

Os CEs são dispositivos alimentados por bateria que contêm um líquido que se torna aerossol quando aquecido, fornecendo nicotina aos usuários com menos irritação do que os cigarros combustíveis (CC). Os CEs também são conhecidos como E-cigs e canetas vape e são um tipo de sistema eletrônico de liberação de nicotina. E-líquidos podem conter uma combinação de propilenoglicol ou bases de glicerina, compostos aromatizantes, nicotina e/ou outras substâncias psicoativas, como derivados de cannabis. Esses dispositivos foram projetados e comercializados por sua capacidade de fornecer nicotina mais rápido do que os concorrentes CC e podem fornecer níveis particularmente elevados de nicotina (Becker \& Rice, 2021).

Em 2019, uma doença respiratória aguda, misteriosa e mortal relacionada à vaporização surgiu, principalmente em pacientes jovens, nos EUA. Os casos aumentaram dramaticamente e atingiram o pico no final de setembro de 2019. O Centro para Controle e Prevenção de Doenças (CDC) denominou a doença que causa esta epidemia de cigarro eletrônico ou lesão pulmonar associada ao uso de produto vaporizador (EVALI). Antes do EVALI, a vaporização tinha sido associada a uma variedade de apresentações pulmonares diferentes, variando de pneumonia lipoide a hemorragia alveolar difusa, mas em números baixos (Chatham-Stephens et al., 2019).

Milhares de usuários de cigarros eletrônicos, predominantemente homens com idade entre 13 e 34 anos, desenvolveram sintomas respiratórios, gastrointestinais e sistêmicos após a vaporização. Testes de e-líquidos vaporizados e amostras das vias aéreas das pessoas afetadas encontraram acetato de vitamina E. Quando o acetato de vitamina E é aquecido às temperaturas altas, ele se decompõe no gás ceteno altamente tóxico. Quando testado em animais, o mesmo causou lesão pulmonar aguda quando inalado por meio de aerossóis de cigarro eletrônico, confirmando-o como o provável produto químico responsável pelo EVALI. Embora haja evidências que associam o acetato de vitamina E a muitos casos de EVALI, existem outros substâncias adulterantes que provavelmente são responsáveis em um subconjunto de casos (Smith, Gotway, Alexander \& Hariri, 2020).

O presente estudo tem como objetivo descrever as consequências e lesões do uso de cigarros eletrônicos.

\section{Metodologia}

Trata-se de um estudo de abordagem qualitativa, onde se adotou a revisão integrativa da literatura, que conforme Galvão (2012), é uma construção de uma análise ampla da literatura com passos pré-definidos uma vez que ela contribui para o 
processo de sistematização e análise dos resultados, visando a compreensão de determinado tema, a partir de outros estudos independentes. Realizado através da Biblioteca Virtual de Saúde (BVS) nas bases de dados do Pubmed e Medline, usando os cruzamentos dos descritores em inglês "Electronic cigarette", "Consequences", "Vaping". Para a avaliação do problema de pesquisa e sua estratificação foi utilizada a estratégia PVO (População/ Problema, Variável/Resultados e Outcomes/ Desfechos) sendo formulada a seguinte estratégia que pode ser observada no Quadro 1. A estratégia supracitada permitiu formular a seguinte questão norteadora: Quais as consequências e lesões do uso de cigarros eletrônicos? A partir da questão norteadora foram utilizados os operadores booleanos para a sistematização das buscas com o seguinte esquema: electronic cigarette AND consequences AND vaping.

Quadro 1. Estratificação do problema de pesquisa seguindo estratégia PVO para formulação de pesquisa.

\begin{tabular}{|c|c|}
\hline $\begin{array}{c}\mathrm{P} \\
\text { População }\end{array}$ & Indivíduos afetados pelo uso de cigarro eletrônico. \\
\hline $\begin{array}{c}\mathrm{V} \\
\text { Variáveis }\end{array}$ & Lesões causadas pelo uso de cigarro eletrônico. \\
\hline $\begin{array}{c}\text { O } \\
\text { Desfechos }\end{array}$ & eletrônico. \\
\hline
\end{tabular}

Fonte: Autores (2021).

Para a seleção dos artigos foram utilizados os seguintes critérios de inclusão: artigos escritos na língua inglesa e portuguesa; publicados entre os anos 2016 até o primeiro semestre de 2021 e que abordem as lesões que o cigarro eletrônico causam. No que diz respeito aos critérios de exclusão, dispensaram-se artigos que se distanciavam da temática central desta revisão e trabalhos que não apresentassem resumos na íntegra nas bases de dados pesquisadas. Após a pré-leitura e leitura seletiva dos textos, foram selecionados 20 artigos (Quadro 2), nos quais realizou-se uma leitura interpretativa buscando responder à pergunta de pesquisa desta revisão.

\section{Resultados e Discussão}

A partir das buscas realizadas nas bases de dados, foi encontrado um total de 308 artigos, sendo selecionados 20 artigos, destes 15 foram encontrados na base de dados Pubmed e 5 na Medline. A seguir, apresenta-se o Quadro 2 que mostra a distribuição dos artigos segundo o título, autores, objetivo, abordagem do artigo, revista e ano.

Quadro 2. Distribuição dos artigos segundo o título, autores, objetivo, abordagem do artigo, revista e ano.

\begin{tabular}{|c|c|c|c|c|c|}
\hline TÍTULO & AUTORES & OBJETIVO & ABORDAGEM & REVISTA & $\begin{array}{c}\text { ANO DE } \\
\text { PUBLICAÇÃO }\end{array}$ \\
\hline $\begin{array}{l}\text { Youth vaping: a review and } \\
\text { update on global } \\
\text { epidemiology, physical and } \\
\text { behavioral health risks, and } \\
\text { clinical considerations }\end{array}$ & $\begin{array}{l}\text { Timothy D. } \\
\text { Becker \& Timothy R. } \\
\text { Rice. }\end{array}$ & $\begin{array}{l}\text { Fornecer aos pediatras e outros médicos } \\
\text { que trabalham com jovens uma pesquisa } \\
\text { com enfoque clínico dos principais } \\
\text { resultados de pesquisas e considerações } \\
\text { com base em evidências recentes. }\end{array}$ & Epidemiologia & $\begin{array}{l}\text { European } \\
\text { Journal of } \\
\text { Pediatrics }\end{array}$ & 2021 \\
\hline $\begin{array}{l}\text { E-cigarettes and Vaping: A } \\
\text { Global Risk for Adolescents }\end{array}$ & $\begin{array}{c}\text { Swati Y Bhave, Nicholas } \\
\text { Chadi }\end{array}$ & $\begin{array}{l}\text { Revisar os diferentes tipos de dispositivos } \\
\text { de vaporização e as substâncias contidas } \\
\text { nos líquidos dos cigarros eletrônicos. }\end{array}$ & Clínica & $\begin{array}{l}\text { Indian } \\
\text { Pediatr }\end{array}$ & 2021 \\
\hline $\begin{array}{l}\text { e-Cigarette Cessation: } \\
\text { Content Analysis of a Quit } \\
\text { Vaping Community on } \\
\text { Reddit }\end{array}$ & Struik L, Yang Y. & $\begin{array}{l}\text { Entender como os usuários de cigarros } \\
\text { eletrônicos estão experimentando e se } \\
\text { aproximando da cessação de } \\
\text { vaping. Especificamente, examinamos os } \\
\text { métodos usados para abordar o abandono, } \\
\text { os motivos para o abandono e as barreiras } \\
\text { e facilitadores para o abandono. }\end{array}$ & Análise Clínica & $\begin{array}{l}\text { J Med } \\
\text { Internet } \\
\text { Res }\end{array}$ & 2021 \\
\hline $\begin{array}{l}\text { Effects of tobacco cigarettes, } \\
\text { e-cigarettes, and waterpipe } \\
\text { smoking on endothelial } \\
\text { function and clinical }\end{array}$ & $\begin{array}{l}\text { Thomas Münzel, Omar } \\
\text { Hahad, Marin Kuntic, } \\
\text { John F Keaney, Jr, John } \\
\text { E Deanfield, e Andreas }\end{array}$ & $\begin{array}{l}\text { Visão geral atualizada do impacto do fumo } \\
\text { de tabaco / narguilé (shisha) e vaping de } \\
\text { cigarro eletrônico na função endotelial, um } \\
\text { biomarcador para aterosclerose precoce, }\end{array}$ & Clínica & Eur Heart J. & 2020 \\
\hline
\end{tabular}




outcomes
$\begin{aligned} & \text { Vaping implications for } \\ & \text { children and youth }\end{aligned}$
Characteristics of
Hospitalized and
Nonhospitalized Patients in a
Nationwide Outbreak of E-
cigarette, or Vaping, Product
Use-Associated Lung Injury
- United States, November
2019

Understanding the
implications of the "vaping
epidemic" among
adolescents and young
adults: A call for action
The electronic cigarette
epidemic in youth and young
adults: A practical review
An updated overview of e-
cigarette impact on human
health

Vaping-related lung injury. Virchows Archiv

Impact of Electronic
Cigarettes on Various Organ
Systems

Perceptions, symptoms, and practices of electronic cigarette users: Descriptive analysis and validation of Arabic short form vaping consequences questionnaire. Review of Health Consequences of Electronic Cigarettes and the Outbreak of Electronic Cigarette, or Vaping, Product UseAssociated Lung Injury.

E-Cigarette or Vaping Product Use-associated Lung Injury: Developing a Research Agenda. An NIH Workshop Report.
Daiber.

Meghan Gilley, Suzanne Beno

Kevin Chatham-

Stephens, Katherine

Roguski, Yunho Jang,

Pyone Cho, Tara C.

Jatlaoui, Sarah Kabbani, Emily Glidden, Emily N.

Ussery, Katrina F.

Trivers, Mary E. Evans, Brian A. King, Dale A.

Rose, Christopher M.

Jones, Grant Baldwin,

Lisa J. Delaney, Peter

Briss, Matthew D. Ritchey.

Nicholas Chadi, Scott E Discutir algumas das pesquisas mais

Hadland, Sion K Harris recentes sobre cigarros eletrônicos,

Brian Burt, Jing Li

Marques, P., Piqueras, L. \& Sanz, MJ

Smith, M. L., Gotway, M. B., Crotty Alexander, L. E., \& Hariri, L. P.

Adam EM Eltorai, Ariel R Choi and Ashley Szabo Eltorai.

Karasneh, R., Al-Azzam, S., Nusair, M., \&

Hawamdeh, S.

Cao, D. J., Aldy, K.,

Hsu, S., McGetrick, M., Verbeck, G., De Silva, I., \& Feng, S. Y.

Laura E. Crotty

Alexander, Lorraine B.

Ware, Carolyn S. Calfee, Sean J. Callahan,

Thomas Eissenberg,

Carol Farver, Maciej L. Goniewicz, Ilona Jaspers, Farrah Kheradmand, Talmadge

E. King, Jr., Nuala J.

Meyer, Vladimir B.

Mikheev, Peter G.

Shields, Alan Shihadeh, Robert Strongin, Robert Tarran

subclínica, de estudos em humanos e animais.

Esta revisão explora como os cigarros eletrônicos funcionam, suas implicações para a saúde, epidemiologia entre os jovens e as estratégias regulatórias atuais. Fornecer dados atualizados sobre as características demográficas dos pacientes e substâncias usadas em produtos de cigarro eletrônico ou vaporização entre pacientes hospitalizados e não hospitalizados, bem como características clínicas observadas entre pacientes não hospitalizados, de acordo com casos relatados ao CDC com dados de entrevista disponíveis, prontuário médico dados de abstração, ou ambos em 5 de novembro de 2019.

Analisar os componentes da vaporização, prevalência, reações adversas, regulamentação, tratamento e estratégias de prevenção relacionadas à vaporização em jovens e adultos jovens.

Realizar um balanço das principais descobertas neste campo e suas consequências para a saúde humana, incluindo a doença coronavírus 2019 (COVID-19).

Discutir os sistemas eletrônicos de entrega de nicotina, bem como a etiologia, apresentação clínica, achados de imagem, características patológicas, tratamento e consequências de longo prazo do EVALI

Revisar os efeitos dos cigarros eletrônicos em vários sistemas fisiológicos, examinamos a associação entre o uso de cigarros eletrônicos e a ingestão e cessação de cigarros combustíveis e destacamos as pesquisas necessárias para construir um consenso.

Investigar as práticas e percepções dos usuários de cigarros eletrônicos na Jordânia e examinar os sintomas (por exemplo, respiratórios) que eles associam ao uso de cigarros eletrônicos.

\section{Descrever os fundamentos dos cigarros} eletrônicos, resumimos os riscos estabelecidos de vaporização, ilustramos as características dos pacientes com EVALI, discutimos o (s) agente (s) causador (es) potencial (is) no surto e apresentamos os tratamentos propostos.

Discutir as complexidades do surto de

lesão pulmonar associada ao uso de cigarro eletrônico ou vaporização (EVALI), para obter uma melhor compreensão de quais devem ser as prioridades de pesquisa, para determinar relações de causa e efeito para

diagnosticar, tratar e prevenir esta doença de forma mais eficaz. estratégias de pesquisa e para descobrir
Clínica

Subst Abus.

\begin{tabular}{c|c}
$\begin{array}{c}\text { Características } \\
\text { clínicas }\end{array}$ & $\begin{array}{c}\text { Curr Opin } \\
\text { Pediatr. }\end{array}$ \\
\hline $\begin{array}{c}\text { Características } \\
\text { clínicas. }\end{array}$ & $\begin{array}{c}\text { MMWR } \\
\text { Morb } \\
\text { Mortal } \\
\text { Wkly Rep. }\end{array}$ \\
\hline
\end{tabular}

Manifestações

JAAPA clínicas \begin{tabular}{c|c}
$\begin{array}{c}\text { Manifestações } \\
\text { clínicas. }\end{array}$ & $\begin{array}{c}\text { Respiratory } \\
\text { Research }\end{array}$
\end{tabular}

Clínica

Internationa 1 journal of pathology

Clínico-patológico Respiratory Care March

\begin{tabular}{c|c}
$\begin{array}{c}\text { Características } \\
\text { Clínicas }\end{array}$ & $\begin{array}{c}\text { Journal of } \\
\text { medical } \\
\text { toxicology }\end{array}$
\end{tabular}

Manejo clínico

Am J 


\begin{tabular}{|c|}
\hline $\begin{array}{l}\text { The Evidence of Electronic } \\
\text { Cigarette Risks Is Catching } \\
\text { Up With Public Perception }\end{array}$ \\
\hline $\begin{array}{l}\text { Short halt in vaping modifies } \\
\text { cardiorespiratory parameters } \\
\text { and urine metabolome: a } \\
\text { randomized trial }\end{array}$ \\
\hline $\begin{array}{l}\text { Prevalence of Electronic } \\
\text { Cigarette Dependence } \\
\text { Among Youth and Its } \\
\text { Association With Future Use }\end{array}$ \\
\hline $\begin{array}{l}\text { Passive exposure of non- } \\
\text { smokers to E-Cigarette } \\
\text { aerosols: Sensory irritation, } \\
\text { timing and association with } \\
\text { volatile organic compounds }\end{array}$ \\
\hline $\begin{array}{l}\text { Vaping epidemic in US } \\
\text { teens: problem and solutions. }\end{array}$ \\
\hline $\begin{array}{l}\text { Understanding Rising } \\
\text { Electronic Cigarette Use. }\end{array}$ \\
\hline
\end{tabular}

\section{Stanton A Glantz}

Martin Chaumont,

Vanessa Tagliatti, El

Mehdi Channan, Jean-

Marie Colet, Alfred

Bernard, Sofia Morra,

Guillaume Deprez, Alain

Van Muylem, Nadia

Debbas, Thomas

Schaefer, Vitalie Faoro,

Philippe van de Borne

Erin A Vogel, Junhan

Cho, Rob S McConnell,

Jessica L Barrington-

Trimis, Adam M

Leventhal

Anna Tzortzi, Stephanie

Teloniatis, George

Matiampa, Gerasimos

Bakelas, Chara Tzavara,

Vergina Konstantina

Vyzikidou, Constantine

Vardavas, Panagiotis

Behrakis, Esteve Fernandez

Venkata, Anand N.;

Palagiri, Raga Deepak R;

Vaithilingam, Siddharthan

Wylie, Blair J;

Hauptman, Marissa;

Hacker, Michele R;

Hawkins, Summer S.
Descrever a evidência de riscos de
cigarros eletrônicos está acompanhando a
percepção pública. Avaliar os efeitos agudos da vaporização e sua reversibilidade nos parâmetros cardiorrespiratórios biológicos / clínicos [pneumoproteínas séricas / urinárias, parâmetros hemodinâmicos, teste de função pulmonar e capacidades de difusão, tensões gasosas transcutâneas (desfecho primário) e fluxo sanguíneo microcirculatório cutâneo].

Examinar a prevalência transversal e a apresentação dos sintomas da dependência de cigarros eletrônicos e determinar se a dependência de cigarros eletrônicos está associada a padrões de uso de cigarros eletrônicos subsequentes 6 meses depois entre os jovens com uso inicial de cigarros eletrônicos no ano anterior.

O presente estudo examinou sintomas de irritação relatados por não fumantes expostos passivamente aos aerossóis de cigarros eletrônicos e seu tempo e associação com as concentrações de compostos orgânicos voláteis produzidos.

Avaliar os fatores que levam ao aumento da vaporização, as razões para sua notável popularidade entre os adolescentes norteamericanos, as consequências da vaporização para a saúde e as medidas para mitigar a epidemia de vaporização.

Compreender o uso crescente do cigarro eletrônico.

\begin{tabular}{|c|c|c|}
\hline Fatores de risco & $\begin{array}{c}\text { JAMA } \\
\text { Netw Open }\end{array}$ & 2019 \\
\hline Clínico-patológico & $\begin{array}{c}\text { Am J } \\
\text { Physiol } \\
\text { Lung Cell } \\
\text { Mol } \\
\text { Physiol }\end{array}$ & 2020 \\
\hline Manejo clínico & $\begin{array}{c}\text { JAMA } \\
\text { Netw Open. }\end{array}$ & 2020 \\
\hline $\begin{array}{l}\text { Características } \\
\text { Clínicas }\end{array}$ & $\begin{array}{l}\text { Environ } \\
\text { Res }\end{array}$ & 2020 \\
\hline $\begin{array}{l}\text { Características } \\
\text { clínicas }\end{array}$ & $\begin{array}{l}\text { Curr Opin } \\
\text { Pulm Med }\end{array}$ & 2021 \\
\hline $\begin{array}{l}\text { Características } \\
\text { clínicas }\end{array}$ & $\begin{array}{l}\text { Obstet } \\
\text { Gynecol }\end{array}$ & 2021 \\
\hline
\end{tabular}

Fonte: Autores (2021).

Os danos do CE à saúde cardiovascular mostram que os consumidores dos dispositivos estão mais expostos ao infarto agudo do miocárdio e doenças cerebrovasculares. O consumo de CE tem sido associado ao aumento da resistência das vias aéreas periféricas e da fração de óxido nítrico exalado, com características semelhantes aos efeitos da fumaça do tabaco, e à lesão pulmonar associada ao uso de produtos de CE. Além disso, a maioria dos jovens usuários de CE relata o uso de politabaco. Embora os aerossóis EC pareçam menos citotóxicos do que os compostos inalados durante o uso do CC, eles apresentam seus próprios riscos à saúde física, especialmente no sistema respiratório. Os CEs podem expor os usuários a metais pesados de baterias e bobinas de aquecimento que podem ser cancerígenos ou tóxicos para o coração e os pulmões, embora os efeitos de longo prazo dessas exposições entre os vapers permaneçam obscuros (Chatham-Stephens et al., 2019; Yang \& Youjin, 2021).

A América do Norte sofreu um surto de lesão pulmonar aguda associada a CE ou vaping (EVALI). Dezenas de mortes e milhares de casos de comprometimento respiratório agudo devido a lesão aguda inespecífica ocorreram. EVALI foi mais intimamente associado à vaporização de derivados de cannabis com acetato de vitamina E, que foi encontrado presente na grande maioria das amostras de lavagem broncoalveolar em uma série de casos de pacientes com EVALI no entanto, outros componentes patogênicos também provavelmente contribuíram. Os achados radiográficos em adolescentes com EVALI incluem nódulos centrolobulares em vidro fosco e opacidades em vidro fosco com preservação subpleural (Struik \& Yang, 2021; Becker \& Rice, 2021; Wylie, Hauptman, Hacker \& Hawkins, 2021).

Em pacientes que apresentam EVALI, os principais sintomas incluem falta de ar, tosse, dor no peito, diarreia, dor 
abdominal, febre e fadiga. Os sintomas ocorrem em qualquer lugar de horas a semanas antes da apresentação. Os exames laboratoriais comumente revelam uma elevada taxa de hemossedimentação e nível de proteína $\mathrm{C}$ reativa, transaminite e leucocitose. Para atender aos critérios do CDC para um caso EVALI "confirmado", os pacientes devem ter vaporizado dentro de 90 dias antes do início dos sintomas, ter infiltrados bilaterais na imagem do tórax, ter uma avaliação negativa para infecção e nenhum outro diagnóstico alternativo plausível (Alexander et al., 2020; Venkata, Palagiri \&Vaithilingam, 2021).

Uma incidência mais alta de hipoxemia pode ser esperada, já que a vaporização do líquido do cigarro eletrônico isoladamente foi associada a alterações nas trocas gasosas pulmonares, além de febre subjetiva, calafrios e fadiga (Chaumont $e t$ al., 2020). Sintomas gastrointestinais também tem sido uma queixa principal comum, sendo náuseas e vômitos os mais comuns, mas podendo apresentar dor abdominal geral e diarreia. Achados incidentais de opacidades pulmonares em vidro fosco na tomografia abdominal obtida durante a investigação de sintomas abdominais primários também podem ocorrer (Vogel, Cho, McConnell, Barrington-Trimis \& Leventhal, 2020; Cao et al., 2020).

A fumaça passiva de cigarros combustíveis difere do aerossol de cigarro eletrônico na gênese e nos constituintes. Os cigarros combustíveis geram fumaça que é principalmente de material sólido e semissólido, enquanto o cigarro eletrônico gera um aerossol semilíquido. Um recente estudo cruzado não cego de exposição a aerossol de segunda mão encontrou aumento na frequência de sintomas de irritação respiratória, falta de ar e dores de cabeça. Além da exposição a 1,8\% de nicotina e aeros sol de CE prejudicar o desenvolvimento alveolar e impactar negativamente o ganho de peso (Tzortzi et al., 2020). Embora o estudo sugira consequências negativas para a saúde do aerossol de CE de segunda mão, são necessários estudos de longo prazo e estudos comparativos com a fumaça de cigarro de segunda mão.

Riscos de saúde adicionais relacionados à vaporização também foram descritos. Vaporizar e ingerir e-líquidos tem sido associado a convulsões. Os CEs também têm um impacto adverso na saúde bucal, embora possivelmente menos severamente do que o CC (Gilley \& Beno, 2020). As baterias usadas nos CEs ainda forma associadas a queimaduras térmicas, queimaduras alcalinas (de hidróxido de lítio), incêndios domésticos e fatalidades foram relatadas pelo seu uso. A pandemia de COVID-19, os adolescentes podem violar o distanciamento social e risco de exposição viral mediado por avaliação neurobiologicamente determinada dos riscos: o compartilhamento de dispositivos de vaporização e o uso sem máscara aumenta o risco de transmissão, aumentando o risco de morbidade para a família no retorno ao lar (Karasneh, Al-Azzam, Nusair \& Hawamdeh, 2021). Além de apresentar riscos para o cérebro em desenvolvimento, lesões pulmonares agudas e efeitos de saúde mental de longo prazo (Bhave \& Chadi, 2021).

Embora os níveis de tóxicos e carcinógenos conhecidos tendam a ser significativamente mais baixos em cigarros eletrônicos do que em fumaça de cigarro combustível, os tóxicos em cigarros eletrônicos líquidos e aqueles que se formam como parte do processo de vaporização podem produzir consequências adversas à saúde por direito próprio. Evidências crescentes disponíveis sobre o potencial carcinogênico do aerossol do cigarro eletrônico (por exemplo, compostos de carbonila e benzeno) e sobre a desregulação de genes associados ao câncer (Glantz et al., 2019). Efeitos agudos foram observados nos sistemas pulmonar, cardiovascular e imunológico, que destacam a necessidade de pesquisas sobre a exposição de longo prazo. Os constituintes químicos específicos e os níveis desses constituintes que apresentam danos permanecem em grande parte não caracterizados (Eltorai, Choi \& Eltorai, 2019).

Os cigarros eletrônicos, com seu alto teor de nicotina, sabores atraentes, baixo custo, ampla disponibilidade e designs discretos, ameaçam 5 décadas de progresso na luta contra o uso do tabaco. Além do risco aumentado de uso subsequente de cigarros tradicionais, maconha, opioides e outras drogas ilícitas, a construção de evidências indica que o uso de cigarro eletrônico também expõe os jovens a vários riscos de saúde agudos e de longo prazo que superam em muito o potencial ainda infundado benefícios do uso de cigarros eletrônicos como uma ferramenta de redução ou cessação do tabagismo nessa faixa etária (Chadi, Hadland, Harris, 2019). 


\section{Conclusão}

A fumaça do cigarro tem efeitos colaterais cardiovasculares graves, levando à disfunção endotelial, aumento do estresse oxidativo e aumento da morbidade e mortalidade cardiovascular. Em geral, o aumento do uso de cigarros eletrônicos é preocupante. Portanto, uma vez que a causa ou causas específicas de EVALI ainda não são conhecidas, a única maneira de as pessoas garantirem que não estão em risco é considerar a abstenção de uso de todos os produtos de cigarro eletrônico, ou vaporização, enquanto esta investigação continua. Em particular, porque a maioria dos pacientes com EVALI relata o uso de produtos contendo antes do início dos sintomas sendo recomendado que as pessoas não usem cigarros eletrônicos ou vaporizadores, especialmente aqueles adquiridos de fontes informais, como amigos, familiares ou revendedores presenciais ou online. Nos últimos anos, adolescentes estão iniciando precocemente o uso de CE, causando preocupação em decorrência da associação entre o CE com o consumo subsequente de cigarros. São necessários programas, políticas e regulamentações de prevenção ao tabaco que dificultem a obtenção de CE pelos jovens.

Os cigarros eletrônicos têm designs elegantes, sabores desejáveis e aceitabilidade social com segurança percebida entre os jovens. Isso resultou no uso epidêmico do CE na juventude, resultando em problemas de saúde significativos de curto e longo prazo. A legislação deve incluir regulamentações que evitem estritamente o marketing e as vendas para jovens, além de reduzir o acesso a esses produtos. Os cigarros eletrônicos e seus tóxicos liberados parecem prejudiciais a vários sistemas orgânicos, embora o corpo de evidências atual seja limitado. Mais pesquisas são necessárias com foco em nas suas consequências, constituintes do e-líquido, características do usuário e padrões de uso.

\section{Referências}

Alexander, L. E. C., Ware, L. B., Calfee, C. S., Callahan, S. J., Eissenberg, T., Farver, C., Goniewicz, M. L., Jaspers, I., Kheradmand, F., King, Jr., T. E., Meyer, N. J., Mikheev, V. B., Shields, P. G., Shihadeh, A., Strongin, R. \& Tarran, R. (2020). Relatório do workshop do NIH: E-cigarro ou uso de produto vaporizador lesão pulmonar associada (EVALI): desenvolvendo uma agenda de pesquisa. Am J Respir Crit Care Med. 202(6): 795-802.

Becker, T. D., Rice, T. R. (2021). Youth vaping: uma revisão e atualização sobre epidemiologia global, riscos para a saúde física e comportamental e considerações clínicas. Eur J Pediatr. 1-10.

Bhave, S. Y \& Chadi, N. (2021). E-cigarettes and Vaping: A Global Risk for Adolescents. Indian Pediatr. 58(4):315-319.

Burt, B. \& Li, J. (2020). The electronic cigarette epidemic in youth and young adults: A practical review. JAAPA. 33(3):17-23

Cao, D. J., Aldy, K., Hsu, S., McGetrick, M., Verbeck, G., De Silva, I., \& Feng, S. Y. (2020). Review of Health Consequences of Electronic Cigarettes and the Outbreak of Electronic Cigarette, or Vaping, Product Use-Associated Lung Injury. Journal of medical toxicology, 16(3), 295-310.

Chadi, N., Hadland, S. E. \& Harris, S. K. (2019). Understanding the implications of the "vaping epidemic" among adolescents and young adults: A call for action. Subst Abus. 40(1):7-10.

Chatham-Stephens, K., Roguski, K., Jang, Y., Cho, P., Jatlaoui, TC, Kabbani, S., Glidden, E., Ussery, EN, Trivers, KF, Evans, ME, King, BA, Rose, DA, Jones, CM, Baldwin, G., Delaney, LJ, Briss, P., Ritchey, M. D. (2019). Características de pacientes hospitalizados e não hospitalizados em um surto nacional de cigarro eletrônico, ou Vaping, lesão pulmonar associada ao uso do produto - Estados Unidos, novembro de 2019. MMWR. Relatório semanal de morbidade e mortalidade, 68 (46), 1076-1080.

Chaumont, M., Tagliatti, V., Channan, E. M., Colet, J., Bernard, A., Morra, S., Deprez, G., Muylem, A. V., Debbas, N., Schaefer, T., Faoro, V. \& Borne, P. V. (2020). Short halt in vaping modifies cardiorespiratory parameters and urine metabolome: a randomized trial. Am J Physiol Lung Cell Mol Physiol. 318(2): L331-L344.

Eltorai, A.E.M., Choi, A.R. \& Eltorai, A. S. (2019) Impact of Electronic Cigarettes on Various Organ Systems. Respiratory Care March, 64 (3) 328-336.

Glantz, S. A. (2019). The Evidence of Electronic Cigarette Risks Is Catching Up With Public Perception. JAMA Netw Open. 2(3):e191032.

Gilley, M. \& Beno, S. (2020). Vaping implications for children and youth. Curr Opin Pediatr. 32(3):343-348.

Karasneh, R., Al-Azzam, S., Nusair, M., \& Hawamdeh, S. (2021). Perceptions, symptoms, and practices of electronic cigarette users: Descriptive analysis and validation of Arabic short form vaping consequences questionnaire. PloS one, 16(1), e0245443.

Marques, P., Piqueras, L. \& Sanz, MJ (2021). An updated overview of e-cigarette impact on human health. Respir Res, 151.

Münzel, T., Hahad, O., Kuntic, M., Jr, J. F. K., Deanfield, J. E. \& Daibere1, A. (2020) Effects of tobacco cigarettes, e-cigarettes, and waterpipe smoking on endothelial function and clinical outcomes. Eur Heart J. 41(41): 4057-4070. 
Research, Society and Development, v. 10, n. 16, e25101623137, 2021

(CC BY 4.0) | ISSN 2525-3409 | DOI: http://dx.doi.org/10.33448/rsd-v10i16.23137

Smith, M. L., Gotway, M. B., Crotty Alexander, L. E., \& Hariri, L. P. (2021). Vaping-related lung injury. Virchows Archiv, 478(1), 81-88.

Struik L., Yang Y. (2021). E-Cigarette Cessation: Content Analysis of a Quit Vaping Community on Reddit, J Med Internet Res, 23 (10).

Tzortzi, A., Teloniatis, S., Matiampa, G., Bakelas, G., Tzavara, C., Vyzikidou, V. K., Vardavas, C., Behrakis, P. \& Fernandez, E. (2020). Passive exposure of non-smokers to E-Cigarette aerosols: Sensory irritation, timing and association with volatile organic compounds. Environ Res. 182:108963.

Venkata, A. N., Palagiri, R. D. R. \& Vaithilingam, S. (2021). Vaping epidemic in US teens: problem and solutions. Curr Opin Pulm Med. $27(2): 88-94$.

Vogel, E. A., Cho, J., McConnell, R. S., Barrington-Trimis, J. L. \& Leventhal, A. M. (2020). Prevalence of Electronic Cigarette Dependence Among Youth and Its Association With Future Use. JAMA Netw Open. 3(2):e1921513.

Wylie, B. J., Hauptman, M., Hacker, M. R. \& Hawkins, S. S. (2021). Understanding Rising Electronic Cigarette Use. Obstet Gynecol. $137(3)$ : $521-527$. 